\title{
Secondary Haematological Cancers in Adults: A Single Centre Experience
}

\author{
Vinila Belum Reddy*, Vanajakshi and Jaya Bhaskar Reddy
}

Department of Pathology, Apollo Institute of Medical Sciences and Research, Hyderabad. India

\section{ABSTRACT}

Introduction: Advances in early detection and treatment mean that more and more people are surviving cancer today. Increased long-term survival seen in patients with solid and hematologic cancers achieved as a result of aggressive chemo radiotherapy has come at a price. Some cancer survivors may develop a new, unrelated cancer later. This is called a second cancer. Reasons for second cancers are varied. Field cancerization, shared environment, familial syndromes, Radiation and Chemotherapy are among the few risk factors affecting the risk of secondary cancers. Therapy-related acute myeloid leukaemia and secondary non-Hodgkin lymphoma has been frequently documented in these patient cohorts. We aim to study the prevalence as well as analyse the factors involved in secondary haematological cancers in our subset of patients.

Methods: This Cross sectional descriptive study was taken up in Apollo Institute of Medical Sciences and Research, Hyderabad. This is a 5 year observational study. We analysed all patients presenting to the Department of pathology, Apollo Hospitals during the period 2010 to 2015.

Results: Total 4 cases of Second cancers were documented in our study. Out of which 2 cases were acute myeloid leukaemias and both of them had history of treatment with alkylating agents. The other 2 cases were diagnosed to have secondary Multiple myeloma. Of the patient presenting with multiple myeloma one of them had history of prior Renal cell carcinoma

Conclusion: Assessment of the risk of second leukaemia should become part of any therapeutic plan for cancer patients. Chemo is known to be a greater risk factor then radiation. Avoidance of drugs with more leukemogenic potential will reduce the occurrence of second leukaemias. It is also important to understand the possibility of a correlation between renal cell carcinoma and multiple myeloma. Field cancerization and increased cytokine expression probably could play an important role in these second cancers.

Keywords: Second primary malignancy, Second cancers, Second Leukaemia's, Secondary haematological cancers

\section{Introduction}

Advances in early detection and treatment mean that more people are surviving cancer today. Increased long term survival seen in patients with solid and hematologic cancers - as a result of aggressive chemo-radiotherapy has come at a price. Some cancer survivors may develop a new unrelated cancer later - called as second cancer. Second cancer is a second neoplasm which differs anatomically, histologically and genetically from the primary neoplasm. Second primary cancers have become an increasingly important concern in oncology during the last two decades, as they now comprise the sixth most common group of malignancies after skin, prostate, breast, lung, and colorectal cancers. ${ }^{[1,2]}$

Survivors of all cancers are living for longer periods, partly because of the more frequent use of effective therapy. The formerly unacceptable toxicities of therapy are more readily controlled with better supportive care. Other reasons for an increase in multiple cancers include fewer competing causes of mortality and consequently more naturally occurring cancers in an increasingly aging population.
Reasons for second cancers are varied. Field cancerisation, Shared environment, Familial syndromes, Radiation and Chemotherapy are among the few risk factors affecting the risk of secondary cancers. ${ }^{[1,2,3,4,5]}$

Secondary Cancers are known to result from the radiation therapy and chemotherapy used to treat primary cancers. The Childhood Cancer Survivor Study, a cohort of more than 14,000 childhood cancer survivors with detailed exposure data and long-term follow-up, has substantially contributed to our understanding of the roles of radiotherapy and chemotherapy in second cancer occurrence. In particular, dose-related risks have been demonstrated for second cancers of the breast, thyroid, central nervous system, gastrointestinal tract, and sarcomas following radiation. Cytotoxic chemotherapy, which has long been known to be leukemogenic, also appears to contribute to risk for a range of other second cancer types. Individuals who develop a second cancer are at particularly high risk for developing additional second cancers. ${ }^{[6]}$

Among adults, risk of second cancers varies substantially by type of first and second cancer, patient age, and 
prevalence of second cancer risk factors, including primary cancer treatments, environmental and lifestyle exposures, and genetic susceptibility. Further research is needed to quantify second cancer risks associated with specific etiologic factors and to identify the patients at highest risk of developing a second cancer to target prevention and screening efforts. Being a tertiary centre, we regularly encounter quite a variety of Haematological cancers. We intend to review and analyse the cases of haematological cancer registered in the last five years in our centre and study the prevalence as well as analyse the factors involved in secondary haematological cancers in our subset of patients.

\section{Materials and Methods}

This Cross sectional descriptive study was taken up in Apollo Institute of Medical Sciences and Research, Hyderabad. This is a 5 year observational study. The present study was done to evaluate the prevalence and pattern of Secondary haematological cancers in Adult patients based upon the haematological specimen received in Department of Haematology, Apollo Institute of Medical Sciences and Research, Hyderabad. All adult patients diagnosed with haematological cancers during the period of 2010-2015 were included in the study. All the cases were reviewed in detail, history and clinical details were retrieved form Hospital medical records and Laboratory electronic records. Data sheet was completed for each patient detailing the Age, Sex, Clinical presentation, History and pertinent Investigations. The various Secondary Haematological cancers were analysed and grouped according the age, sex and presentation. Data was entered into excel data sheet and appropriate statistical analysis was performed.

\section{Results}

From July 2010 to June 2015, total 450 cases were diagnosed to have hematological cancers. Out of them only 4 were diagnosed to have Secondary Hematological Cancers $(2.2 \%$ of cases $)$.

Case I: A 66-year old postmenopausal woman came with a complaint of lower abdominal mass in July 2010, ultrasound detected left adnexal mass with elevated CA125 levels $(366 \mathrm{IU} / \mathrm{ml} / \mathrm{RR}=<21 / \mathrm{U} / \mathrm{ml})$. A total abdominal hysterectomy with bilateral salpingo-oophorectomy, pelvic lymph node dissection and omentectomy were performed. The histopathology diagnosis was poorly differentiated carcinoma. She was staged as FIGO stage IIIb and submitted to six courses of systemic chemotherapy with paclitaxel and carboplatin (total cumulative dose-1200mg and $2700 \mathrm{mg}$ respectively). Following completion of chemotherapy course in October 2010, her CA-125 levels were normalized with normal haematological parameters. Computed tomography and Magnetic resonance imaging showed no residual diseases. Based on these findings she achieved in remission and was followed with routine surveillance. Following 27 months of disease free survival, she was admitted on February 2013 with anaemia and received 2units of blood transfusion. Bone marrow aspiration and biopsy revealed Myelodysplastic Syndrome (MDS) with trilineage dysplasia. FISH analyses on bone marrow aspirate showed $5 \mathrm{q} 31$ and $7 \mathrm{q} 31$ deletion whereas a mutation of JAK2V617F was present by qualitative PCR analysis. Based on these findings a diagnosis of therapy related MDS/MPN-U was rendered. Subsequently 6 months later leukemic transformation with Acute Myeloid Leukaemia (AML) -M4 was noted. Patient refused for further treatment and expired within 2 week's time.

\begin{tabular}{|c|c|c|c|c|c|} 
Ovarian cancer stage IIIb & \multicolumn{2}{c}{ Onset of MDS/MPN-U } & Onset of AML-M4 & Expired \\
\hline $\begin{array}{c}\downarrow \\
\text { July } 2010\end{array}$ & 2011 & 2012 & February 2013 & 6 August 2013 & 22 August 2013 \\
\hline
\end{tabular}

Case II: A 30year old male patient presented with Burkitts Lymphoma (February 2008). He was evaluated and was treated with Cyclophosphamide and Cytarabine based chemotherapy with which he achieved remission and was on follow up. Subsequently in on follow up he went on to develop Acute Leukaemia (AML M5) in June 2013. He went on to develop second cancer 5 years after achieving remission from Burkitts Lymphoma.

Case III: A 66 Year old male patient presented with case of Papillary Renal Cell carcinoma (RCC). He underwent Left partial Nephrectomy (14/6/11). Subsequently he went on to develop symptoms of easy fatigability associated with anaemia and deranged renal parameters in January 2014. On evaluation he was diagnosed to have Multiple Myeloma (MM). He developed this inspite of not receiving any chemotherapy.

Case IV: A 55year old female patient is a case of Carcinoma Breast. She underwent Radical Mastectemy in March 2007. Post surgery she received Chemotherapy (Cytarabine + Adriamycin + 5 FU + Cyclophosphamide). Later she went on to develop Multiple myeloma in November 2014, for which she underwent Autologous Stem cell transplant.

Out of the 4 cases diagnosed to have Secondary Haematological cancers, 3 of them had history of Cytotoxic 
Chemotherapy. One patient developed Secondary Haematological cancer, in spite of not receiving any chemotherapy or radiotherapy. None of them received any radiotherapy.

Among those developing Secondary Haematological cancers secondary to Chemotherapy, one patient received carboplatin based Chemotherapy and two patients received Alkylating agents (Cyclophosphamide) based Chemotherapy. Among the patients receiving Alkylating agent one patient developed AML-M5 and other developed multiple myeloma. The patient who received Carboplatin based chemotherapy developed MDS which progressed to AML-M4.

\section{Discussion}

Therapy-related myeloid neoplasm's (t-MN) are noted in approximately 10 to $20 \%$ of all cases of AML, MDS \& MDS/MPN. ${ }^{[3,6,7]}$ More than $50 \%$ of patients with secondary AML have breast cancer, Non Hodgkin's Lymphoma (NHL), and Hodgkin's Disease(HD). In the present study, 3 out of 4 cases $(75 \%)$ diagnosed as Secondary Haematological cancers had history of prior chemotherapy. Both the patients (2/4) who developed secondary acute myeloid leukaemias received Chemotherapy.

Some types of chemotherapy (chemo) drugs have been linked with different kinds of cancer. The cancers most often linked to chemo are Myelodysplastic syndrome (MDS) and

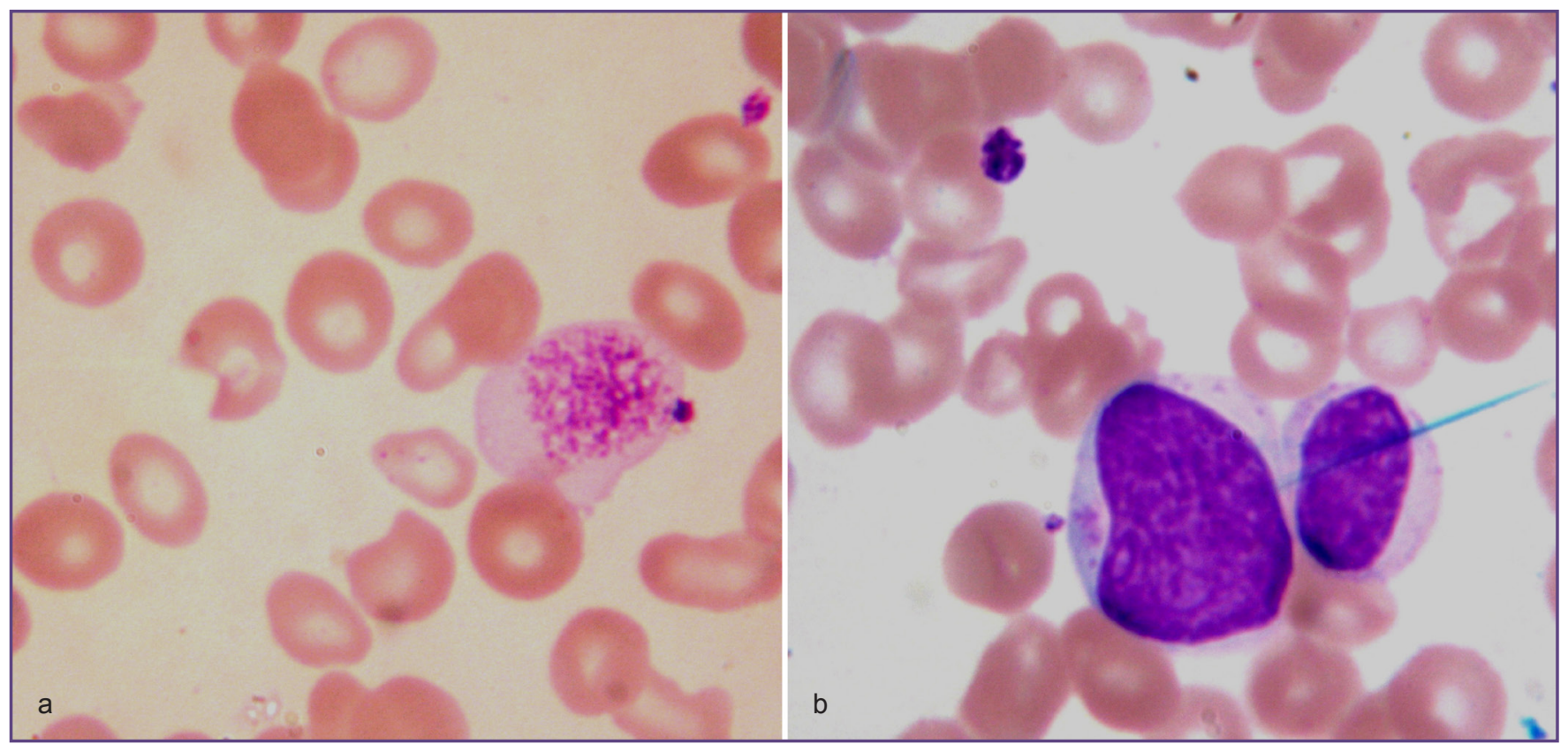

Fig. 1: (Case I) Peripheral smear shows MDS with trilineage dysplasia; (1a) Macroovalocytes with macrohypogranular dyspoietic platelets; (1b)Blast \& hypogranular metamyelocyte (Giemsa stain, x100)

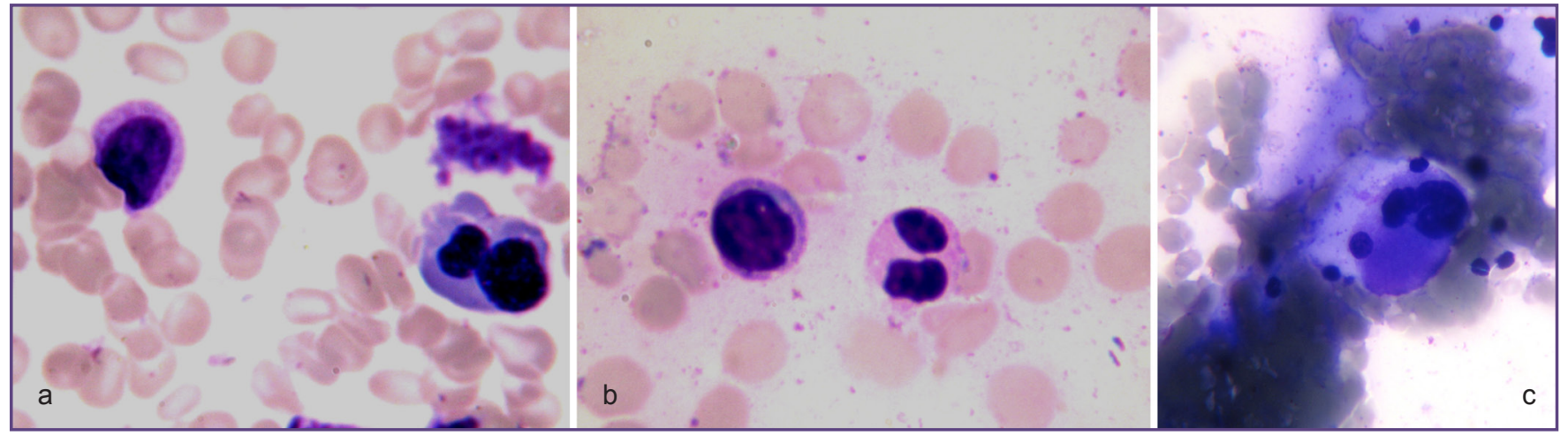

Fig. 2: (Case I) Bone marrow aspiration cytology shows MDS with trilineage dysplasia (a) Binucleate normoblast (b) Pelgeroid neutrophil with hypogranular myelocyte (c) Micromegakaryocyte with hypolobated partially detached nucleus (Giemsa stain, x100) 


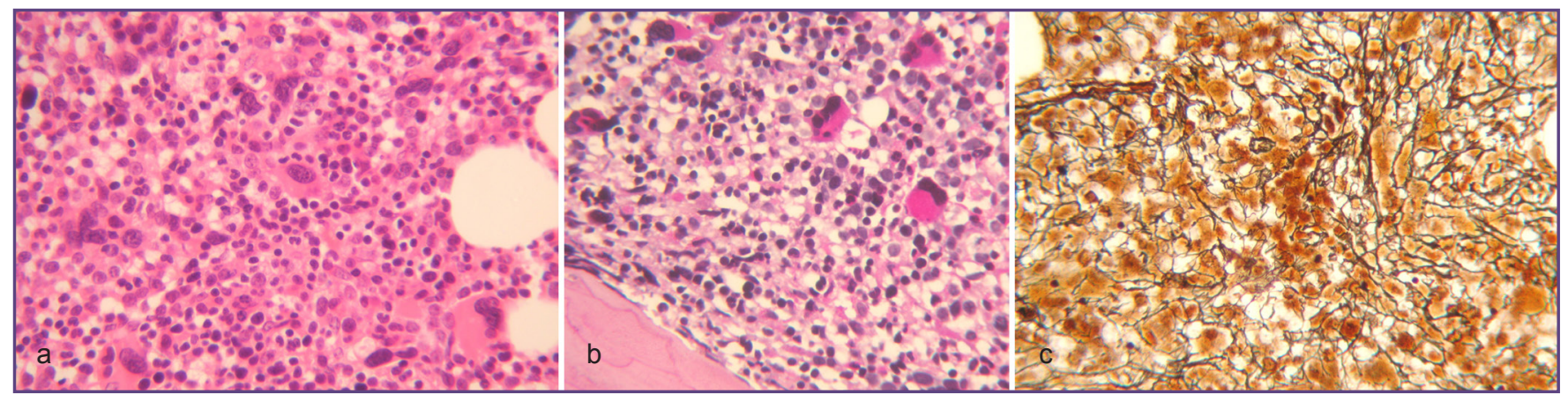

Fig. 3: (Case I) Bone marrow biopsy shows MDS with trilineage dysplasia (a)Hyperplastic and dyspoietic erythropoiesis, hyperplastic granulopoiesis with prominence of blasts, hyperplastic megakaryoctes with hypolobated micromegakaryocytes; (H and E, x400) (b)Hypolobated micromegakaryocytes, (PAS, x400) (c) Grade 3 on scale of 0-3(Reticulin, x400).

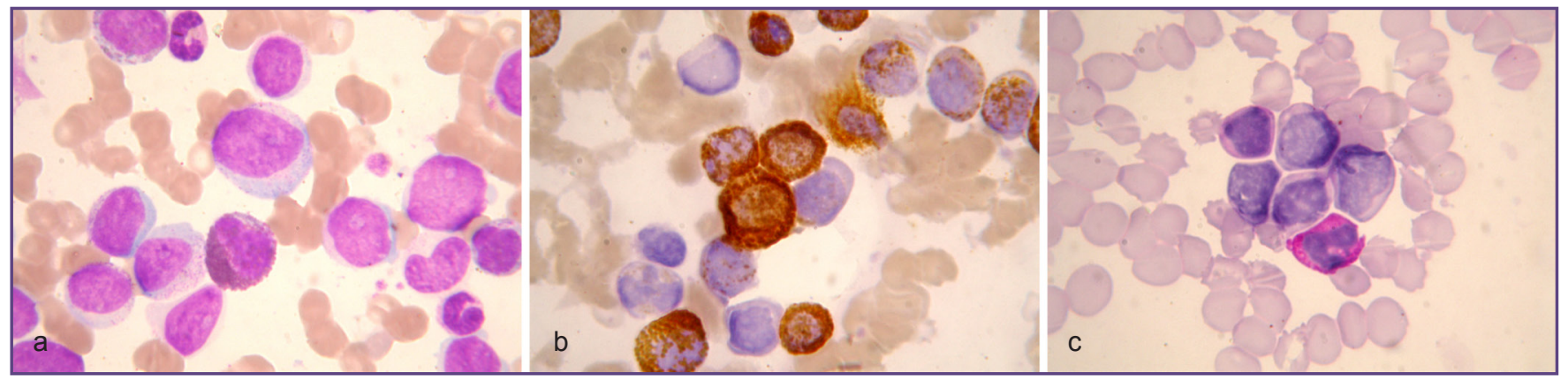

Fig. 4: (Case I) Peripheral smear shows acute leukaemic transformation (a)Immature leucocytes with prominence of blasts (Giemsa stain, $\mathrm{x100)(b)Some} \mathrm{of} \mathrm{the} \mathrm{blasts} \mathrm{show} \mathrm{myeloperoxidase} \mathrm{granules} \mathrm{(MP0,} \mathrm{x100)(c)Blasts} \mathrm{show} \mathrm{negative} \mathrm{staining} \mathrm{on}$ PAS (PAS, x100).

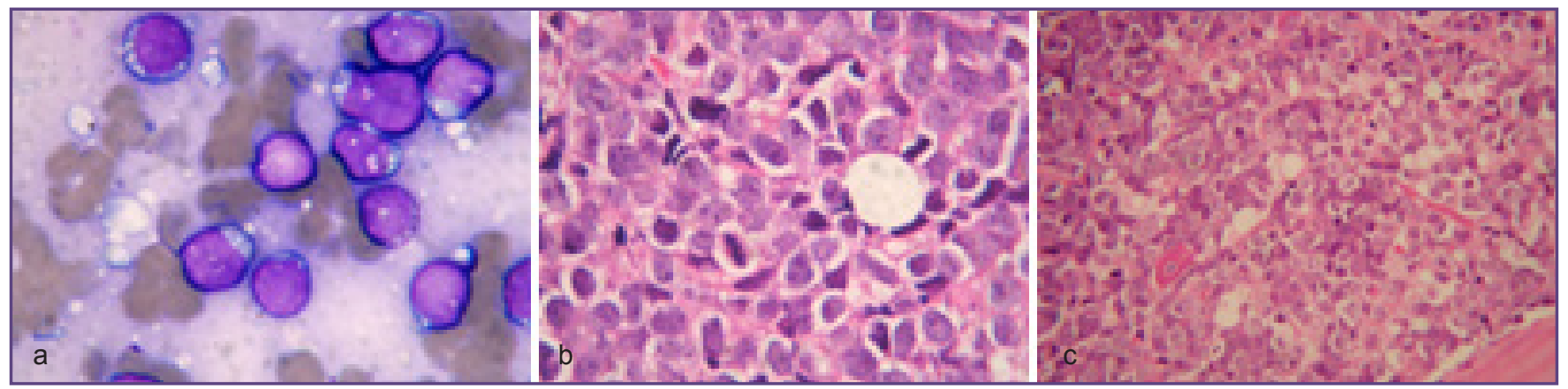

Fig. 5: (Case II) (a) Peripheral smear shows sheets of Monoblasts in high power field (Giemsa stain, x100) (b) \& (c) Bone marrow biopsy sections showing blasts with increased mitotic activity (high and low power).

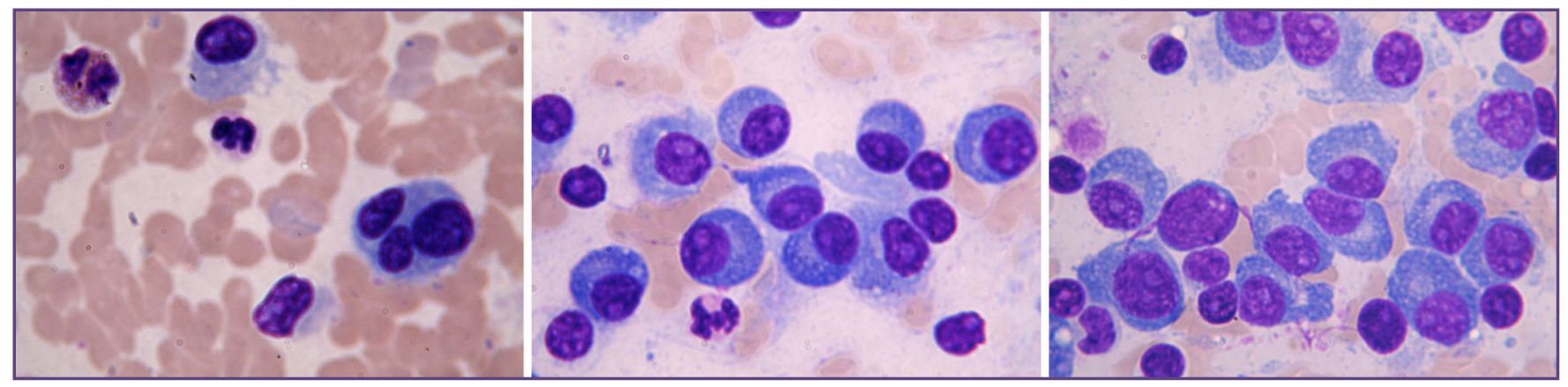

Fig. 6: (Case III): Bone marrow aspirate sections showing sheets of plasmaytoblasts .(high and low power). 
acute Myelogenous leukaemia (AML). Sometimes, MDS occurs first, and then turns into AML. Acute lymphocytic leukaemia (ALL) has also been linked to chemo. Chemo is known to be a greater risk factor than radiation therapy in causing leukaemia. Alkylating agents, nitrosureas and procarbazine appear to have the highest leukemogenic potential. ${ }^{[6,8]}$

1. Alkylating Agents:

a. Mechlorethamine

b. Chlorambucil

c. Cyclophosphamide (Cytoxan)

d. Melphalan

e. Lomustine (CCNU)

f. Carmustine (BCNU)

g. Busulfan

2. Topoisomerase II inhibitors

a. Etoposide

b. Teniposide

c. Mitoxantrone

d. Anthracyclines

i. Doxorubicin (Adriamycin)

ii. Daunorubicin

iii. Epirubicin

iv. Idarubicin

3. Others

a. Cisplatin

b. Carboplatin

One of the patient who developed AML-M5 received Alkylating agent (Cyclophosphamide) and another Patient who developed MDS which progressed to AML-M4 received Cisplatin based chemotherapy. Alkylating agents are chemo drugs that interfere with a cell's DNA in a certain way. These drugs can sometimes cause AML and MDS. Often MDS develops first, which then progresses to AML. Alkylating agents related leukaemias are similar to post MDS leukaemia's with preleukemic phase and trilineage dysplasia. 5 and 7 chromosomal cytogenetic abnormalities are seen, with a poor prognosis. The risk gets higher with higher drug doses, longer treatment time, and higher dose-intensity (more drugs given over a short period of time). Studies have shown that leukaemia risk begins to rise about 2 years after treatment with alkylating agents, becomes highest after 5 to 10 years, and then declines. Unfortunately, MDS and leukaemia that develop after treatment with alkylating agents tend to be hard to treat and have a poor outcome with a median survival of 8 months. ${ }^{[2,3,6,8]}$
The chemo drugs cisplatin and carboplatin are not alkylating agents, but they attack cancer cells in much the same way. These drugs seem to increase the risk of leukaemia (mainly AML), too, but the risk is not as great as with the alkylating agents. This leukaemia is hard to treat and tends to have a poor outcome, much like the leukaemia linked to the alkylating agents. The risk of leukaemia rises as the amount of drug used gets higher. The risk of developing leukaemia increases even more if radiation is given along with cisplatin or carboplatin. Both the patients in our study had poor outcomes with early mortality. $[2,3,6,8]$

The class of chemo drugs called topoisomerase II inhibitors stop cells from being able to repair DNA. These drugs can also cause leukaemia, mainly AML. Leukaemia develops sooner after treatment with these drugs than the leukaemia from alkylating agents. Most cases are found within 2 or 3 years of treatment and without MDS occurring first. Balanced translocations involving chromosome 11 q23 are usually seen. Leukaemia from topoisomerase II inhibitors tends to respond to better to treatment and has a better outlook than the leukaemia from alkylating agents. Drugs called anthracyclines are also topoisomerase II inhibitors. Anthracyclines are less likely to cause leukaemia than the other topoisomerase II inhibitors. ${ }^{[6,8]}$

Most kinds of leukaemia, including AML, CML and ALL can be caused by past radiation exposure. Myelodysplastic syndrome (MDS), a bone marrow cancer that can turn into acute leukaemia, has also been linked to past radiation exposure. The risk of these diseases after radiation treatment depends on a number of factors such as:

1. How much of the bone marrow was exposed to radiation

2. The amount of radiation that reached the bone marrow

3. The radiation dose rate (how much was given in each dose, how long it took to give the dose, and how often it was given)

The person's age when they were treated with radiation does not seem to be a risk factor. Most often, these cancers develop within several years of radiation treatment, peaking at 5 to 9 years after exposure. ${ }^{[2,3,6,8]}$ None of the patients in our study had an history of prior radiotherapy. This could be due to the referral bias.

Multiple myeloma is a hematologic disorder characterized by monoclonal proliferation of plasma cells in the bone marrow that secrete immunoglobulin's. For the diagnosis of myeloma it is necessary to detect $>10 \%$ plasma cells in bone marrow or tissue biopsy. Its incidence is approximately 3-4/100.000 and responsible for about $1 \%$ of all malignancy related deaths. Not uncommonly 
this is seen as a second cancer. ${ }^{[9,10]}$ In the present study two cases $(2 / 4-50 \%)$ were diagnosed to have secondary Multiple myelomas.

One patient developed Multiple myeloma after chemotherapy for Breast carcinoma (Alkylating agent, Cyclophosphamide). In the literature there are reports of Multiple myeloma cases secondary to chemotherapy and it has long been recognized. ${ }^{[9,10,11]}$ Marinopoulos et al reported a case of M.M after chemotherapy for non small cell lung cancer (cisplatin based). ${ }^{[1]}$ Radiotherapy and alkylating agents used in the treatment of carcinoma breast pose the risk of myelodysplasia and secondary leukaemia's. Plasma cell dyscrasias, plasmacytoma and multiple myeloma have rarely been reported in the literature coexisting with carcinoma breast. ${ }^{[10]}$ Multiple myeloma was reported in 5 out of 443 second neoplasms after treatment for Breast cancer in a study by Levi $\mathrm{F}$ et al. ${ }^{[7]}$

Another developed M.M after partial nephrectomy for papillary renal cell carcinoma, with history of neither Radiation nor Chemotherapy. These tumours may represent coincidence of two not so uncommon tumours in elderly population or may show a true association. ${ }^{[13]}$ Number of case reports and a small case series has hypothesized an association between renal cell carcinoma (RCC), and multiple myeloma (MM). ${ }^{[14,15,16,17]}$ This hypothesis has been confirmed in a large population-based study from the USA and the association is supposed to be bidirectional, pointing to shared risk factors rather than treatment related factors. Patients with RCC have higher relative risk of developing MM during follow-up and vice versa. ${ }^{[18]}$

Although not necessarily specific to this association, certain genetic risk factors may be shared between renal cell carcinoma and multiple myeloma that contribute to the observed bidirectional association. For example, $c$-met oncogene mutations are well recognized in hereditary papillary renal cell carcinoma. ${ }^{[19,20]}$ Hereditary papillary renal cell carcinoma is most often observed in older age, an attribute that is consistent with multiple myeloma incidences. More importantly, c-met expression was recently implicated in myeloma cell proliferation through feedback loops with interleukin-6, an inflammatory cytokine that has a critical role in the development and growth of myeloma cells. ${ }^{[20]}$ Therefore, $c$-met expression may be a candidate for elucidating the association between renal cell carcinoma and multiple myeloma.

Lifestyle-related risk factors such as obesity may also contribute to the observed association. Obesity is a strong risk factor for renal cell carcinoma and has modest but consistent effects on multiple myeloma incidences. [21, 22] Furthermore, obesity is characterized by marked changes in adipose tissue, such as increased number and size of adipocytes. Adipose tissue is a major source of inflammatory mediators, particularly interleukin-6. [23] Therefore, obesity may facilitate the creation of a microenvironment that supports the development of renal cell carcinoma and multiple myeloma. ${ }^{[23,24,25]}$

Shared environmental risk factors are another factor which plays important role in second cancers. Sometimes the second cancer isn't nearby, but is still linked to the same cancer-causing agent. Lifestyle factors, such as smoking, alcohol, exercise, sun exposure, and diet, clearly play individual roles in a long list of cancers, such as those of the head and neck, lung, bladder, skin and gastrointestinal tract, which are often seen as secondary cancers following treatment for a primary neoplasm. Environmental risk factors continue to raise the probability for a new cancer after the diagnosis and treatment of an initial malignancy. $[2,3,5,26]$

For some cancers, having that cancer means you are at an increased risk of getting another cancer in the same organ or nearby. This may be because the whole organ (and sometimes nearby organs and tissues) were exposed to the same cancer-causing agents that led to the first cancer. This means that the entire area could already have early changes that can lead to cancer. This is called field cancerization. Although cancer survivors often reduce their exposures, the effects of earlier exposure can continue to influence their risks for years. Individuals with a genetic predisposition to multiple neoplasm's are another group at risk. Polymorphisms for metabolizing enzymes are another potentially critical category of etiologic factors that cannot yet be evaluated. ${ }^{[26]}$

\section{Conclusion}

Assessment of risk of secondary leukemia should be a part of therapeutic plan for cancer patients. Chemotherapy is a greater risk factor than radiation. Avoidance of drugs with more leukomegenic potential will be beneficial. Correlation between Renal cell carcinoma and Multiple myeloma needs to be further studied. Shared risk factors, Field cancerisation and increased cytokine expression could probably play an important role in these cancers.

\section{References}

1. Ng AK, Travis LB. Subsequent malignant neoplasms in cancer survivors. Cancer J. 2008;14(6):429-34.

2. Travis LB. The epidemiology of second primary cancers. Cancer Epidemiol Biomarkers Prev.2006;15.

3. Howlader N, Noone A, Krapcho M, et al. SEER Cancer Statistics Review, 1975-2010. National Cancer Institute. http://seer.cancer.gov/csr/ 1975_2010/. Accessed April 2013. 
4. Siegel R, DeSantis C, Virgo K, et al. Cancer treatment and survivorship statistics. CA Cancer J Clin. 2012; 62:220-241.

5. Morton LM, Chanock SJ. A step toward slaying the hydra of second cancers. Nat Med. 2011;17:924-925.

6. Robison LL, Armstrong GT, Boice JD, et al. The Childhood Cancer Survivor Study: a National Cancer Institutesupported resource for outcome and intervention research. $J$ Clin Oncol. 2009;27:2308-2318.

7. Levi F, Te VC, Randimbison L, et al: Cancer risk in woman with previous breast cancer. Ann Oncol 2003;14:71-73.

8. Curtis RE, Boice JD Jr, Stovall M, et al: Risk of leukemia after chemotherapy and radiation treatment for breast cancer. N Engl J Med 1992;326:1745-1751.

9. Tomono H, Fujioka S, Kato K, Yoshida K, Nimura Y. Multiple myeloma mimicking bone metastasis from breast cancer: report of a case. Surg Today. 1998;28(12):1304-6.

10. Pruneri, G., Ginieri, S., Peccatori, F., et al. Plasma Cell Myeloma Coexisting With Metastatic Breast Carcinoma in the Bone Marrow. J Clin Oncol 2005;23:232-233.

11. Marinopoulos S, Skorda L, Karatapanis S, Rasidakis A. Multiple myeloma emerging after chemotherapy for nonsmall cell lung cancer. Med Oncol 2008; 25:415-418.

12. Hingmire S, Hingmire S, Bakshi A, Gujaral S, Badwe R, Nair R. Multiple myeloma mimicking bone metastasis in a patient of carcinoma breast. Indian J Med Paediatr Oncol 2008;29:53-5.

13. Mubarak M. Renal cell carcinoma and plasma cell myeloma: Coincidence or true association? Urology Annals. 2014;6(3):256-257.

14. Badros A, Karakunnel J, Dawson N. Multiple myeloma and renal cell carcinoma possible association. Leuk Lymphoma. 2007;48:1662-4. [PubMed: 17701606]

15. Choueiri TK, Baz RC, McFadden CM, Khasawneh M, Karam MA, Kelly M, et al. An association between renal cell carcinoma and multiple myeloma: A case series and clinical implications. BJU Int. 2008;101:712-5.
16. Ozturk MA, Dane F, Kaygusuz I, Asmaz O, Uzay A, Bayik $\mathrm{M}$, et al. Synchronous renal cell carcinoma and multiple myeloma: Report of two cases and review of the literature. J BUON. 2009;14:511-4.

17. Bhandari MS, Mazumder A, Jagannath S, Vesole DH. Association between renal cell carcinoma and plasma cell dyscrasias: A case series of six patients. Clin Lymphoma Myeloma. 2008;8:188-90. [PubMed: 18650185]

18. Ojha RP, Evans EL, Felini MJ, Singh KP, Thertulien R. The association between renal cell carcinoma and multiple myeloma: Insights from population-based data. BJU Int. 2011;108:825-30.

19. Padhi S, Sahoo PK, Banerjee D, Ghosh RN. Renal cell carcinoma and plasma cell myeloma: unique association and clinical implications. Urol Ann. 2014;6:252-6.

20. Hov H, Tian E, Holien T et al. c-Met signaling promotes IL6-induced myeloma cell proliferation. Eur J Haematol 2009; 82: $277-87$.

21. Alexander DD, Mink PJ, Adami HO et al. Multiple myeloma: a review of the epidemiologic literature. Int J Cancer 2007; 120 (Suppl. 12): 40-61.

22. Moore LE, Wilson RT, Campleman SL. Lifestyle factors, exposures, genetic susceptibility, and renal cell cancer risk: a review. Cancer Invest 2005; 23: 240-55

23. Sakai A, Kawano M, Kuramoto A. Interleukin-6 produced by renal-cell carcinoma cells and progression of multiple myeloma. N Engl J Med. 1991;324:1893-4.[PubMed: 2041555]

24. Eder K, Baffy N, Falus A, Fulop AK. The major inflammatory mediator interleukin-6 and obesity. Inflamm Res 2009; 58: 727-36.

25. Hauner H. Secretory factors from human adipose tissue and their functional role. Proc Nutr Soc 2005; 64: 163-9.

26. Morton, LM, Onel, K, Curtis, RE, Hungate, EA, and Armstrong, GT. The rising incidence of second cancers: patterns of occurrence and identification of risk factors for children and adults. Am Soc Clin Oncol Educ Book. 2014; 2014: e57-e67.

*Corresponding author:

Dr. Vinila Belum Reddy, Assistant Professor, Department of Pathology, Apollo Institute of Medical Sciences and Research, Hyderabad. India Email: vinilabelum@gmail.com

Date of Submission : $\mathbf{3 0 . 0 5 . 2 0 1 7}$

Date of Acceptance : 06.07.2017

Financial or other Competing Interests: None.

Date of Publication : 26.10.2017 